\title{
Fast-kick-off monotonically convergent algorithm for searching optimal control fields
}

\author{
Sheng-Lun Liao, ${ }^{1}$ Tak-San Ho, ${ }^{2}$ Shih-I Chu, ${ }^{1,3}$ and Herschel Rabitz ${ }^{2}$ \\ ${ }^{1}$ Center for Quantum Science and Engineering and Department of Physics, National Taiwan University, Taipei 10617, Taiwan \\ ${ }^{2}$ Department of Chemistry, Princeton University, Princeton, New Jersey 08544, USA \\ ${ }^{3}$ Department of Chemistry, University of Kansas, Lawrence, Kansas 66045, USA
}

(Received 30 June 2011; published 7 September 2011)

\begin{abstract}
This Rapid Communication presents a fast-kick-off search algorithm for quickly finding optimal control fields in the state-to-state transition probability control problems, especially those with poorly chosen initial control fields. The algorithm is based on a recently formulated monotonically convergent scheme [T.-S. Ho and H. Rabitz, Phys. Rev. E 82, 026703 (2010)]. Specifically, the local temporal refinement of the control field at each iteration is weighted by a fractional inverse power of the instantaneous overlap of the backward-propagating wave function, associated with the target state and the control field from the previous iteration, and the forward-propagating wave function, associated with the initial state and the concurrently refining control field. Extensive numerical simulations for controls of vibrational transitions and ultrafast electron tunneling show that the new algorithm not only greatly improves the search efficiency but also is able to attain good monotonic convergence quality when further frequency constraints are required. The algorithm is particularly effective when the corresponding control dynamics involves a large number of energy levels or ultrashort control pulses.
\end{abstract}

DOI: 10.1103/PhysRevA.84.031401

PACS number(s): 32.80.Qk, 33.80.-b, 02.60.Pn, 42.50.Hz

Quantum control problems are generally concerned with finding optimal control fields that maximize some physical objectives. A common quantum control objective is to drive a quantum system from a given initial state to a final state that maximizes the corresponding transition probability [1,2]. In recent years, much progress in the quantum control study has been made by drawing on powerful computers and state-of-the-art laser-pulse-shaping technologies [3], as well as optimal control theory [4-8]. Computationally, two key issues are usually encountered for solving optimal quantum control problems: (1) to find optimal control fields numerically and (2) to impose necessary frequency constraints on the calculated control fields. The former may require numerous iterations for solving the corresponding time-dependent Schrödinger equations, especially when starting with poorly chosen initial control fields (which often occurs when involving large numbers of energy levels or ultrashort control pulses), rendering it computationally formidable, whereas the latter usually requires frequency filtering at the end of each iteration, becoming detrimental to search effort.

The first issue has typically been addressed by invoking various conventional optimization schemes, including conjugategradient and quasi-Newtonian methods [9], and, especially, a class of monotonically convergent algorithms specifically formulated for optimal quantum control problems. The existing monotonically convergent approaches include the well-known Krotov method [10,11], Zhu-Rabitz method [12,13], MadayTurinici method [14], and a recently formulated two-point boundary-value quantum control paradigm (TBQCP) method based on the local control theory [15-17]. These monotonically convergent methods, especially the TBQCP-based schemes [16], allow for much larger search steps throughout iterations and converge superlinearly, in contrast to the usual gradient-based methods $[18,19]$. In practice, the resultant optimal control fields calculated using these algorithms often require further frequency constraint [20,21], which could in turn hinder the convergence rate or even the monotonicity.
Recently, various hybrid schemes [22,23] have been proposed by combining the existing monotonically convergent algorithms and conventional optimization schemes to obtain a quadratic convergence behavior near the asymptotes. This Rapid Communication presents a different scheme, based on the TBQCP algorithm, aiming for fast kick-off of the search efficiency, especially with poorly chosen initial control fields, while effectively preserving the monotonic convergence property when subject to the frequency constraint. Specifically, the local temporal progression of the control field at each iteration is weighted by a fractional inverse power of the instantaneous overlap of the backward-propagating wave function, associated with the target state and the control field from the previous iteration, and the forward-propagating wave function, associated with the initial state and the concurrently refining control field.

The fast-kick-off TBQCP algorithm is implemented via a time-dependent overlap function for the state-to-state transition probability optimal control problems. Within the electric dipole approximation, the evolution of the quantum system in the presence of $E(t)$ is governed by the time-dependent Schrödinger equation

$$
i \hbar \frac{\partial}{\partial t}|\psi(t)\rangle=\left\{H_{0}-\mu E(t)\right\}|\psi(t)\rangle,
$$

where $H_{0}$ and $\mu$ are the unperturbed Hamiltonian and dipole moment of the quantum system, respectively. Here we seek an optimal time-dependent external field $E(t), t \in[0, T]$, that can steer a quantum system from an initial state $|\psi(0)\rangle=|i\rangle$ to a final state $|f\rangle$ of the field-free Hamiltonian $H_{0}$ of the controlled quantum system.

Following the original TBQCP method [16,17], we introduce a dynamical invariant

$$
O(t) \equiv|\chi(t)\rangle\langle\chi(t)|
$$

associated with a reference control field $E^{(0)}(t)$ and the final state $|f\rangle$, where the wave function $|\chi(t)\rangle$ satisfies the 
time-dependent equation

$$
i \hbar \frac{\partial}{\partial t}|\chi(t)\rangle=\left\{H_{0}-\mu E^{(0)}(t)\right\}|\chi(t)\rangle,|\chi(T)\rangle \equiv|f\rangle .
$$

It can then be shown that the positive semidefinite Hermitian operator $O(t)$ is a solution of the invariant equation [24]

$$
\frac{\partial}{\partial t} O(t)+\frac{1}{i \hbar}\left[O(t), H_{0}-\mu E^{(0)}(t)\right]=0, O(T)=|f\rangle\langle f|,
$$

and the expectation value $\left\langle\psi^{(0)}(t)|O(t)| \psi^{(0)}(t)\right\rangle=\langle i|O(0)| i\rangle$ $\forall t \in[0, T]$ is independent of time $t$, where the wave function $\left|\psi^{(0)}(t)\right\rangle$ satisfies the equation

$$
i \hbar \frac{\partial}{\partial t}\left|\psi^{(0)}(t)\right\rangle=\left\{H_{0}-\mu E^{(0)}(t)\right\}\left|\psi^{(0)}(t)\right\rangle
$$

for an arbitrary initial condition $\left|\psi^{(0)}(0)\right\rangle$.

From Eqs. (1) and (4), we derive the following integral equation:

$$
\begin{aligned}
& \langle\psi(T)|O(T)| \psi(T)\rangle-\langle\psi(0)|O(0)| \psi(0)\rangle \\
& =\int_{0}^{T} f_{\mu}(t)\left\{E(t)-E^{(0)}(t)\right\} d t
\end{aligned}
$$

or equivalently

$$
\frac{d}{d t}\langle\psi(t)|O(t)| \psi(t)\rangle=f_{\mu}(t)\left\{E(t)-E^{(0)}(t)\right\},
$$

where

$$
f_{\mu}(t) \equiv-\frac{2}{\hbar} \operatorname{Im}\{\langle\psi(t)|O(t) \mu| \psi(t)\rangle\} .
$$

From Eqs. (4) and (7), and by invoking the local control theory [15-17], we can then choose the following ansatz:

$$
E(t)=E^{(0)}(t)+\eta S(t) \varepsilon_{\alpha}(t)
$$

for the control field $E(t)$, where

$\varepsilon_{\alpha}(t)=\frac{f_{\mu}(t)}{\zeta_{\alpha}(t)}=-\frac{2}{\hbar} \operatorname{Im}\left\{\frac{\langle\psi(t) \mid \chi(t)\rangle}{|\langle\psi(t) \mid \chi(t)\rangle|^{\alpha}} \times\langle\chi(t)|\mu| \psi(t)\rangle\right\}$,

$0 \leqslant \alpha \leqslant 1$. Here the search step parameter $\eta>0$ designates the size of the change from $E^{(0)}(t)$ to $E(t)$, the envelop function $S(t) \geqslant 0$ describes the pulse shape of the control fields, and the overlap function (a positive function)

$$
\zeta_{\alpha}(t) \equiv|\langle\psi(t) \mid \chi(t)\rangle|^{\alpha} \leqslant 1
$$

is a fractional power of the instantaneous overlap $|\langle\psi(t) \mid \chi(t)\rangle|$ of the backward-propagating wave function $|\chi(t)\rangle$, associated with the target state $|f\rangle$ and the control field $E^{(0)}(t)$, and the forward-propagating wave function $|\psi(t)\rangle$, associated with the initial state $|\psi(0)\rangle$ and the concurrently refining control field $E(t)$. Note that although the overlap function $\zeta_{\alpha}(t)$ may vary from 0 (for a zero control, i.e., when $E^{(0)}(t)=0 \forall t \in[0, T]$ ) to 1 [i.e., when $E^{(0)}(t)$ becomes optimal], the ratio

$$
\mathcal{A}_{\alpha}(t) \equiv \frac{\langle\psi(t) \mid \chi(t)\rangle}{|\langle\psi(t) \mid \chi(t)\rangle|^{\alpha}}=\mathcal{A}_{1}(t) \times|\langle\psi(t) \mid \chi(t)\rangle|^{1-\alpha}
$$

is bounded between -1 and 1 , i.e., $\left|\mathcal{A}_{\alpha}(t)\right| \leqslant 1 \quad \forall \alpha \in$ $[0,1]$, where $\mathcal{A}_{1}(t)=\langle\psi(t) \mid \chi(t)\rangle /|\langle\psi(t) \mid \chi(t)\rangle|, \quad\left|\mathcal{A}_{1}(t)\right|=$
$1 \geqslant\left|\mathcal{A}_{\alpha}(t)\right|$, and $0 \leqslant \zeta_{\alpha}(t) \leqslant|\langle\psi(t) \mid \chi(t)\rangle| \leqslant 1 \quad \forall \alpha \in[0,1]$. Thus, assuming that the dipole moment operator $\mu$ is bounded and $\alpha$ lies between 0 and 1, the function $\varepsilon_{\alpha}(t)$, cf. Eq. (10), always remains finite even if $\zeta_{\alpha}(t)$ is very close to zero.

Equations (1), (3), (9), and (10) are the working equations of the fast-kick-off TBQCP method for iteratively refining the control field $E(t)$, starting with an arbitrary reference control field $E^{(0)}(t)$. Asymptotically (i.e., when approaching an optimal control field), $\zeta_{\alpha}(t) \rightarrow 1$ and the new algorithm converges to the original TBQCP method corresponding to the $\alpha=0$ case $[16,17]$. It is not obvious how to choose a proper search-step parameter $\eta$ such that the TBQCP method can be both efficient and stable, especially when intense control fields are involved. Although a large $\eta$ value can quickly increase the target yield in the beginning of the iterations, it can also make the convergence behavior unstable. The implementation of $\zeta_{\alpha}(t)$ can increase the search efficiency without resorting to some artificially large $\eta$ values, thus greatly reducing the likelihood of numerical instability. Specifically, the overlap function $\zeta_{\alpha}(t)$ is adopted to increase (and kick off) the rate change

$$
\frac{d}{d t}\langle\psi(t)|O(t)| \psi(t)\rangle=\eta S(t) \frac{\left[f_{\mu}(t)\right]^{2}}{\zeta_{\alpha}(t)} \geqslant \eta S(t)\left[f_{\mu}(t)\right]^{2} \geqslant 0,
$$

thus leading to improvement of the monotonic property

$$
\begin{aligned}
\langle\psi(T)|O(T)| \psi(T)\rangle & \geqslant\langle\psi(0)|O(0)| \psi(0)\rangle \\
& =\left\langle\psi^{(0)}(T)|O(T)| \psi^{(0)}(T)\right\rangle
\end{aligned}
$$

throughout the iterations, especially when $\zeta_{\alpha}(t) \ll 1$ at the early search stages, for example, as a result of a poor choice of the initial control field $E^{(0)}(t)$.

The resultant control field $E(t)$ may be further filtered to remove the unwanted low- and high-frequency components, for example, in its simplest fashion, by invoking the relation

$$
\mathcal{F}^{-1}\{\mathcal{F}\{E(\cdot)\} h(\omega)\} \rightarrow E(t), t \in[0, T]
$$

at the end of each iteration, where $\mathcal{F}$ and $\mathcal{F}^{-1}$ are, respectively, Fourier and inverse Fourier transforms and $h(\omega)$ is any proper bandpass filter function [16]. In general, filtering out the unwanted frequency components of $E(t)$ is detrimental. In the following calculations, however, we show that the inclusion of the overlap function $\zeta_{\alpha}(t)$ can preserve the monotonic convergence quality of the TBQCP method. Atomic units were used throughout, unless mentioned otherwise.

For comparisons between the new fast-kick-off and original TBQCP methods, two commonly studied control problems were chosen in the numerical simulations: (1) the control of vibrational transitions [13] and (2) the control of ultrafast electron tunneling [22]. For the former, we consider a one-dimensional Morse oscillator with the potential $V(x)=D_{0}\left\{\exp \left[\beta\left(x-r_{0}\right)\right]-1\right\}^{2}-D_{0}$, with the parameters $D_{0}=0.1994, \beta=1.189$, and $r_{0}=1.821$ chosen to mimic a vibrating $\mathrm{OH}$ diatom, and the dipole moment function approximated as $\mu(x)=\mu_{0} x \exp \left(-x / r^{*}\right)$, with $\mu_{0}=$ 3.088 and $r^{*}=0.6$. For the latter, we consider an electron in the one-dimensional asymmetric double-well potential $V(x)=\frac{1}{64} x^{4}-\frac{1}{4} x^{2}+\frac{1}{256} x^{3}$, together with the dipole moment $\mu(x)=-x$. In all calculations, Eqs. (1) and (3), as well 


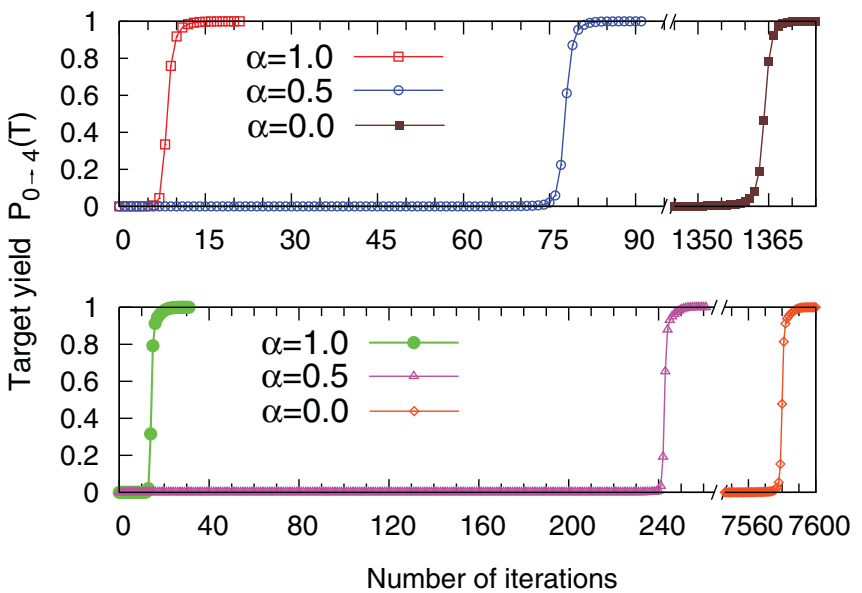

FIG. 1. (Color online) Unfiltered (top) and filtered (bottom) TBQCP convergence history for the $0 \rightarrow 4$ transition at three different $\alpha$ values $0.0,0.5,1.0$ and for the parameter $\eta=0.1$.

as the corresponding eigenstate problems, were solved using the generalized pseudospectral method [25], the pulse-shape function was $S(t)=\sin ^{2}(\pi t / T)$, and the filter function $h(\omega)$ was chosen as the Butterworth bandpass filter [16] of order 50 . All control simulations were started with poorly chosen initial fields and ended with optimal control fields that produce target yields equal to 0.9999 approximately.

Figure 1 shows the unfiltered (top) and filtered (bottom, corresponding to a frequency window $[0.005,0.02]$ in atomic units) TBQCP convergence history at three different $\alpha$ values, $\alpha=0,0.5,1.0$, all starting with the same initial control field (producing an initial target yield of $\approx 10^{-7}$ ), for the $0 \rightarrow$ 4 vibrational transition control simulations of the Morse oscillator using the search-step parameter $\eta=0.1$. It is found that the rate of the fast kick-off increases greatly with the $\alpha$ values (the original TBQCP corresponds to $\alpha=0$ ). Moreover, although the frequency constraint reduces the convergence rate (by $\approx 1.5-, 3-$, and 6-fold for $\alpha=1.0,0.5$, and 0.0 , respectively), the monotonicity is preserved throughout the iterations. Importantly, the filtered results at sufficiently large $\alpha$ values (here $\alpha=0.5,1.0$ ) still converge much faster than their unfiltered counterparts (i.e., at $\alpha=0$ ).

Figure 2 displays the windowed Fourier transform of unfiltered and filtered optimal control fields corresponding to the $\alpha=1$ TBQCP results in Fig. 1. It was found that the

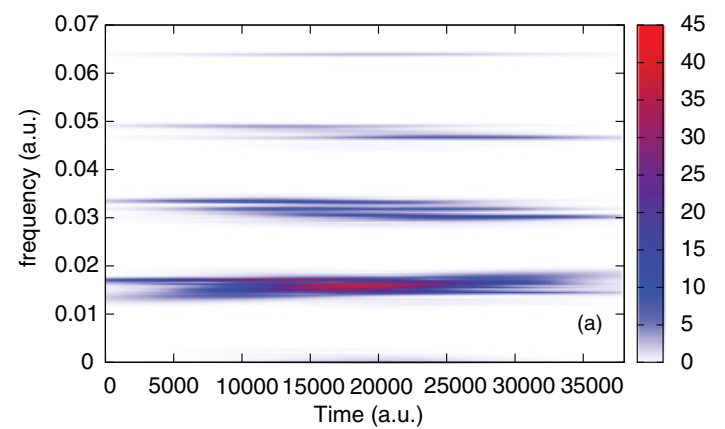

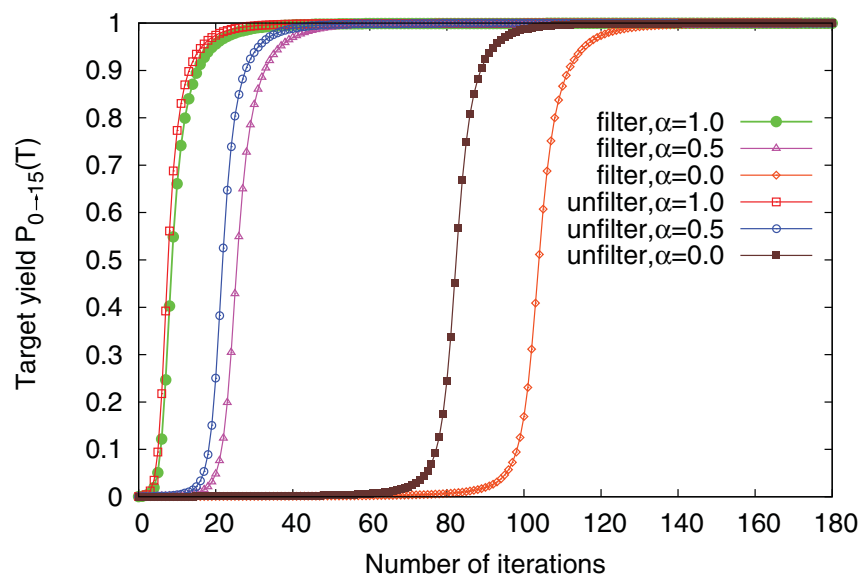

FIG. 3. (Color online) Unfiltered and filtered TBQCP convergence history for the $0 \rightarrow 15$ transition at three different $\alpha$ values, $0.0,0.5$, and 1.0, and for the search-step parameter $\eta=0.1$. The maximum amplitude of optimal control fields is less than 0.026 a.u. and the pulse length $T=50265$ a.u. $(\approx 1.21 \mathrm{ps})$.

dominant frequencies of the filtered optimal field lie mainly between 0.01 and 0.02 a.u., in sharp contrast to the unfiltered one, which contains all significant ones up to 0.07 a.u.

Figure 3 shows the unfiltered and filtered TBQCP convergence behavior (with an initial target yield of $\approx 10^{-4}$ ) at three $\alpha$ values, $0.0,0.5$, and 1.0 , for the $0 \rightarrow 15$ transition control of the Morse oscillator using the search-step parameter $\eta=0.1$.

It is found that, in addition to increasingly faster kick-off rates at larger $\alpha$ values, the unfiltered and filtered (corresponding to a frequency window $[0.005,0.02]$ in atomic units) control simulations also exhibit very similar convergence behavior.

Figure 4 shows the TBQCP convergence history (with an initial target yield of $\approx 10^{-9}$ ) at different $\alpha$ values for the ultrafast tunneling of the electron from the ground state (in the deeper left well) to the first excited state (in the right well) in the asymmetric double well using the search-step parameter $\eta=0.01$.

It is shown that the implementation of the time-dependent overlap function in the TBQCP scheme leads to a much faster kick-off rate when searching ultrashort optimal controls (with the pulse length equal to $T \approx 12 \mathrm{fs}$ ). It is also found (not shown) that the frequency constraint does not have much influence on the monotonic convergence property.

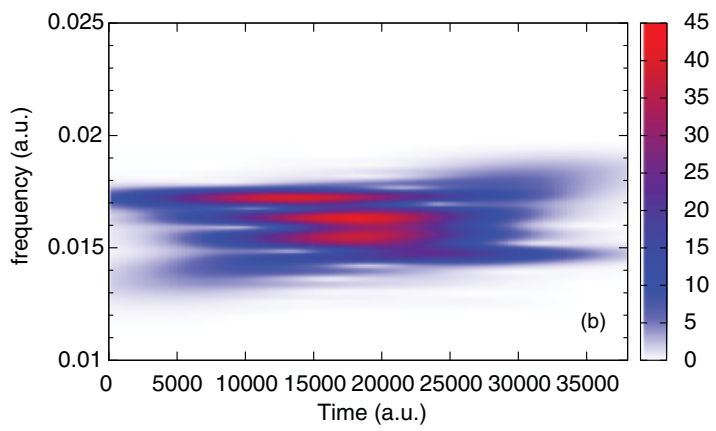

FIG. 2. (Color online) Windowed Fourier transform of (a) unfiltered and (b) filtered optimal fields for the $0 \rightarrow 4$ transition control simulations at $\alpha=1$ in Fig. 1. These windowed Fourier transforms are very similar to each other for frequencies between 0.01 and 0.02 a.u. The maximum amplitude of optimal control fields is less than 0.023 a.u. and the pulse length is $T=37700$ a.u. $(\approx 0.91 \mathrm{ps})$. 

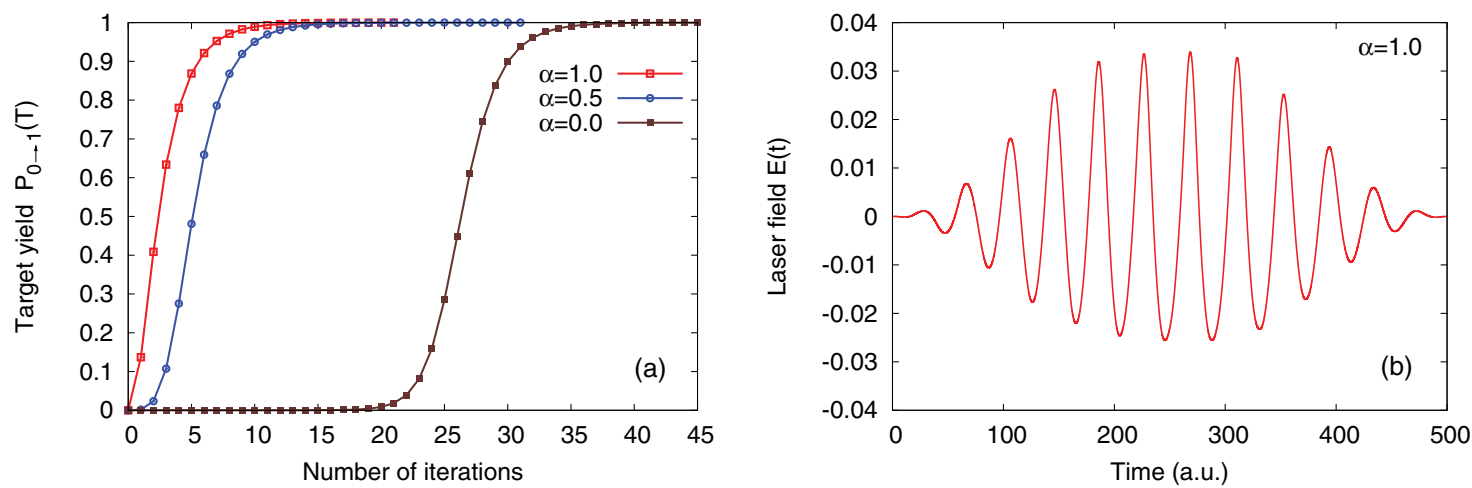

FIG. 4. (Color online) (a) Unfiltered TBQCP convergence history for the $0 \rightarrow 1$ transition (corresponding to the tunneling from the left well to the right one) at three different $\alpha$ values, $0.0,0.5$, and 1.0, and (b) the corresponding optimal field for the $\alpha=1$ case. In all calculations $\eta=0.01$ and the pulse length is $T=502$ a.u. $(\approx 12 \mathrm{fs})$.

In summary, we have presented a fast-kick-off algorithm for searching optimal control fields that maximize the stateto-state transition probability, especially with poorly chosen initial control fields. The new algorithm is based on a recently formulated monotonically convergent iteration scheme, the TBQCP method, aided by the implementation of an instantaneous overlap function that monitors the search progress throughout. Our numerical control simulations for vibrational state-to-state transitions and for ultrafast electron tunneling have demonstrated that the new algorithm not only can greatly improve the search efficiency over its original one, but it also can attain good monotonic convergence quality in the case of the frequency constraints. The new algorithm is especially effective when the underling control dynamics involves a large number of energy levels or ultrashort control pulses, and it should be of particular interest for controlling complicated quantum processes including, for example, photoassociation reactions $[1,2,26]$, molecular isomerization $[27,28]$, and highorder harmonic generation [29-31], for which a judicious choice of sound initial control fields may be difficult.

This work was partially supported by National Science Council of Taiwan (Grant No. 100-2119-M-002-013-MY3), National Taiwan University (Grant No. 10R80700), the US Department of Energy, and the U.S. National Science Foundation.
[1] E. F. de Lima, T.-S. Ho, and H. Rabitz, Phys. Rev. A 78, 063417 (2008).

[2] E. F. de Lima, T.-S. Ho, and H. Rabitz, Chem. Phys. Lett. 501, 267 (2011)

[3] A. M. Weiner, Rev. Sci. Instrum. 71, 1929 (2000).

[4] A. P. Peirce, M. A. Dahleh, and H. Rabitz, Phys. Rev. A 37, 4950 (1988).

[5] S. Shi, A. Woody, and H. Rabitz, J. Chem. Phys. 88, 6810 (1988).

[6] R. Kosloff, S. A. Rice, P. Gaspard, S. Tersigni, and D. J. Tannor, Chem. Phys. 139, 201 (1989).

[7] S. Rice and M. Zhao, Optical Control of Molecular Dynamics (Wiley, New York, 2000).

[8] J. Werschnik and E. K. U. Gross, J. Phys. B 40, R175 (2007).

[9] W. H. Press, S. A. Teukolsky, W. T. Vetterling, and B. P. Flannery, Numerical Recipes in Fortran, 2nd ed. (Cambridge University Press, New York, 1992).

[10] V. F. Krotov, Automat. Remote Control 34, 1863 (1973); 35, 1 (1974); 35, 345 (1974).

[11] J. Somlói, V. A. Kazakov, and D. J. Tannor, Chem. Phys. 172, 85 (1993).

[12] W. Zhu, J. Botina, and H. Rabitz, J. Chem. Phys. 108, 1953 (1998).

[13] W. Zhu and H. Rabitz, J. Chem. Phys. 109, 385 (1998).

[14] Y. Maday and G. Turinici, J. Chem. Phys. 118, 8191 (2003).

[15] M. Mirrahimi, G. Turinici, and P. Rouchon, J. Phys. Chem. A 109, 2631 (2005).

[16] T.-S. Ho and H. Rabitz, Phys. Rev. E 82, 026703 (2010).
[17] T.-S. Ho, H. Rabitz, and S.-I. Chu, Comput. Phys. Commun. 182, 14 (2011).

[18] N. Khaneja, T. Reiss, C. Kehlet, T. Schulte-Herbruggen, and S. J. Glaser, J. Magn. Reson. 172, 296 (2005).

[19] I. I. Maximov, Z. Tosner, and N. C. Nielsen, J. Chem. Phys. 128, 184505 (2008).

[20] C. Gollub, M. Kowalewski, and R. de Vivie-Riedle, Phys. Rev. Lett. 101, 073002 (2008).

[21] M. Lapert, R. Tehini, G. Turinici, and D. Sugny, Phys. Rev. A 79, 063411 (2009).

[22] A. Castro and E. K. U. Gross, Phys. Rev. E 79, 056704 (2009).

[23] R. Eitan, M. Mundt, and D. J. Tannor, Phys. Rev. A 83, 053426 (2011).

[24] H. R. Lewis Jr. and W. B. Riesenfeld, J. Math. Phys. 10, 1458 (1969).

[25] X.-M. Tong and Shih I Chu, Chem. Phys. 217, 119 (1997).

[26] C. P. Koch, R. Kosloff, E. Luc-Koenig, F. Masnou-Seeuws, and A. Crubellier, J. Phys. B 39, S1017 (2006).

[27] M. Artamonov, T.-S. Ho, and H. Rabitz, Chem. Phys. 328, 147 (2006).

[28] Y. Kurosaki, M. Artamonov, T.-S. Ho, and H. Rabitz, J. Chem. Phys. 131, 044306 (2009).

[29] J. L. Krause, K. J. Schafer, and K. C. Kulander, Phys. Rev. Lett. 68, 3535 (1992).

[30] X. Chu and Shih I Chu, Phys. Rev. A 64, 021403(R) (2001).

[31] J. Heslar, D. Telnov, and Shih I Chu, Phys. Rev. A 83, 043414 (2011). 